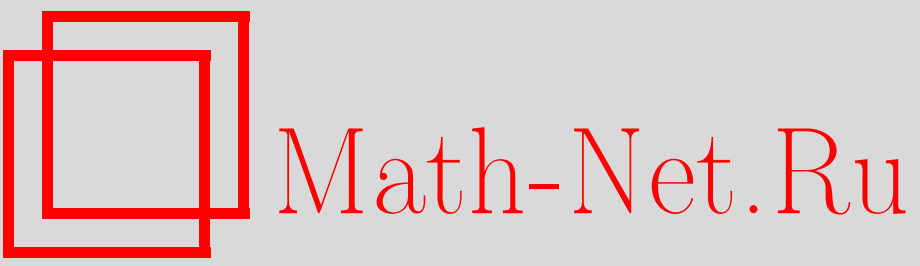

В. А. Попов, Обобщение понятия полноты риманова аналитического многообразия, Итоги науки и техн. Сер. Соврем. мат. и ее прил. Темат. обз., 2020, том $181,84-101$

DOI: https://doi.org/10.36535/0233-6723-2020-181-84-101

Использование Общероссийского математического портала Math-Net.Ru подразумевает, что вы прочитали и согласны с пользовательским соглашением

http://www.mathnet.ru/rus/agreement

Параметры загрузки:

IP: 52.6 .47 .48

26 апреля 2023 г., 17:59:39 


\title{
ОБОБЩЕНИЕ ПОНЯТИЯ ПОЛНОТЫ РИМАНОВА АНАЛИТИЧЕСКОГО МНОГООБРАЗИЯ
}

\author{
(C) 2020 г. $\quad$ В. А. ПОПОВ
}

\begin{abstract}
АннотАция. В работе рассматривается аналитическое продолжение локально заданной римановой метрики. Приведено обобщение понятия полноты, реализуемое как аналитическое продолжение произвольной римановой метрики. Изучены различные римановы метрики, прежде всего, связанные со строением алгебры Ли g всех векторных полей Киллинга для локально заданной метрики. Введено понятие квазиполного многообразия, обладающего свойством продолжаемости всех локальных изометрий до изометрий всего многообразия. Получена классификация псевдополных многообразий малых размерностей. Приведены условия, которым должна удовлетворять алгебра Ли всех векторных полей Киллинга $\mathfrak{g}$ и ее стационарная подалгебра $\mathfrak{h}$ локально однородного псевдориманова многообразия, при которых локально однородное многообразие аналитически продолжается до однородного.
\end{abstract}

Ключевые слова: риманово многообразие, псевдориманово многообразие, алгебра Ли, аналитическое продолжение, векторное поле, группа Ли, замкнутая подгруппа.

\section{GENERALIZATION OF THE NOTION OF COMPLETENESS OF A RIEMANNIAN ANALYTIC MANIFOLD}

\author{
(c) 2020 V. A. POPOV
}

\begin{abstract}
In this paper, we discuss the concept of an analytic continuation of a local Riemannian metric. We propose a generalization of the notion of completeness realized as an analytic continuation of an arbitrary Riemannian metric. Various Riemannian metrics are studied, primarily those related to the structure of the Lie algebra $\mathfrak{g}$ of all Killing vector fields for a local metric. We introduce the notion of a quasicomplete manifold, which possesses the property of extendability of all local isometries to isometries of the whole manifold. A classification of pseudocomplete manifolds of small dimensions is obtained. We present conditions for the Lie algebra of all Killing vector fields $\mathfrak{g}$ and its stationary subalgebra $\mathfrak{h}$ of a locally homogeneous pseudo-Riemannian manifold under which a locally homogeneous manifold can be analytically extended to a homogeneous manifold.
\end{abstract}

Keywords and phrases: Riemannian manifold, pseudo-Riemannian manifold, Lie algebra, analytic continuation, vector field, Lie group, closed subgroup.

AMS Subject Classification: 53C20, 54H15

1. Введение. Рассмотрим риманово аналитическое многообразие $M$ и шар $U \subset M$ малого радиуса с центром в некоторой точке $x_{0} \in M$. Под аналитическим продолжением локально заданной метрики будем подразумевать любое риманово аналитическое многообразие $N$, для которого существует аналитическая изометрия $\varphi: U \rightarrow M$. Поставим задачу найти наиболее естественное аналитическое продолжение данной метрики. Естественным требованием является свойство 
непродолжаемости искомого многообразия, введенного еще в классических монографиях С. Хелгасона [4] и С. Кобаяси, Ш. Номидзу [1]. Однако непродолжаемые многообразия могут быть весьма неестественными, например, односвязная накрывающая правой полуплоскости выколотыми точками $(1 / n, k / n), k, n \in \mathbb{N}$.

В исследованиях по геометрии римановых пространств в целом, как правило, существенным требованием является полнота рассматриваемого многообразия. Для полного односвязного аналитического риманова многообразия любая изометрия $\varphi: U \rightarrow M$ между двумя связными открытыми подмножествами $U \subset M, V \subset M$ аналитически продолжается до изометрии $\varphi: M \rightarrow M$ (см. [4]).

Однако в общем случае шар $U$ риманова аналитического многообразия нельзя изометрически вложить в полное риманово аналитическое многообразие, т.е., вообще говоря, локально заданная риманова метрика аналитически не продолжается до метрики полного риманова многообразия. Возникает вопрос об обобщении понятия полноты. Естественным обобщением такого рода является непродолжаемость риманова аналитического многообразия.

Зададимся вопросом, можно ли по заданным локальным свойствам римановой аналитической метрики, т.е. метрики, заданной на малом шаре $U$, построить риманово аналитическое многообразие $M$, содержащее $U$ в качестве открытого подмножества, и допускающего аналитическое продолжение локальных изометрий до изометрий всего многообразия? Другими словами, любая ли изометрия $\varphi: U \rightarrow V$ между двумя связными открытыми подмножествами $U \subset M$, $V \subset M$ аналитически продолжается до изометрии $\varphi: M \rightarrow M ?$ Непреодолимым препятствием для такого продолжения является следующий факт. Пусть $\mathfrak{g}-$ алгебра Ли всех векторных полей Киллинга на римановом аналитическом многообразии $M$ и $\mathfrak{h} \subset \mathfrak{g}$ - ее стационарная подалгебра: для фиксированной точки $p \in M$ считаем, что $X \in \mathfrak{h}$ тогда и только тогда, когда $X(p)=0$. Пусть $G$ - односвязная подгруппа, порожденная алгеброй $\mathfrak{g}$, и $H$ - ее подгруппа, порожденная подалгеброй $\mathfrak{h}$. Пусть $G$ действует на односвязном многообразии $M$; тогда орбита фиксированной точки $p \in M$ является подмногообразием, изометричным фактор-группе $G / H$. Но фактор-группа $G / H$ является многообразием лишь в случае замкнутости подгруппы $H$ в $G$, а это выполняется не всегда.

Целью данной работы является определение псевдополного многообразия, являющееся «наиболее полным» аналитическим продолжением произвольной локально заданной римановой аналитической метрики. Изучается аналитическое продолжение локально заданной римановой метрики. Рассмотрим случаи вполне неоднородной метрики и метрики, для которой алгебра Ли всех векторных полей Киллинга не имеет центра. В этих случаях дадим определение квазиполного многообразия $M$, обладающего свойством единственности и продолжаемости всех локальных сохраняющих ориентацию изометрий $f: U \rightarrow V$, где $U, V$ - связные открытые подмножества многообразия $M$, до изометрии $f: M \rightarrow M$. Ориентированное риманово аналитическое многообразие, алгебра векторных полей которого имеет нулевой центр, называется квазиполным, если оно непродолжаемо и не допускает нетривиальных сохраняющих ориентацию и все векторные поля Киллинга локальных изометрий в себя. Приведем определение псевдополного многообразия, приводящее к «наиболее полному» продолжению локально заданной метрики и применимое к произвольной локально заданной метрике. Риманово аналитическое односвязное ориентированное многообразие $M$ называется псевдополным, если оно обладает следующими свойствами:

(i) $M$ непродолжаемо;

(ii) не существует локально изометрического сохраняющего ориентацию накрывающего отображения $f: M \rightarrow N$, где $N$ - односвязное риманово аналитическое многообразие, а $f(M)-$ открытое подмножество в $N$, не равное $N$.

Среди псевдополных многообразий выделим «наиболее симметричные» правильные псевдополные многообразия. Далее изучим псевдополные многообразия малых размерностей и приведем их классификацию.

Второй целью является изучение локально однородных многообразий, причем не только римановых, но и псевдоримановых. Далее приведем условия, при которых $H$ замкнута в $G$. Строение 
незамкнутых подгрупп хорошо известно. Однако соответствующие исследования носят чисто алгебраический характер и никак не учитывают локальные свойства римановой метрики. Описание свойств незамкнутой подгруппы $U \subset M$ содержится в классической работе А. И. Мальцева [5]. Если подгруппа Ли $H$ односвязной группы Ли $G$ не замкнута в $G$, то группа $G$ содержит такой тор $T$, что пересечение $H \cap T$ является всюду плотной обмоткой этого тора. Однако этот факт трудно установить исходя из локальных свойств заданной римановой аналитической метрики, т.е. исходя из свойств алгебры Ли $\mathfrak{g}$ и стационарной подалгебры h. Можно ли найти свойства алгебры Ли всех векторных полей Киллинга, при выполнении которых подгруппа $H$, определяемая стационарной подалгеброй $\mathfrak{h}$, будет замкнутой в односвязной группе $G$, порожденной алгеброй $\mathfrak{g}$ ? В этом направлении отметим результат Мостова, согласно которому $H$ замкнута в $G$, если $\mathfrak{h}$ полупроста. Кроме того, Мостов доказал, что $H$ замкнута в $G$, если $\operatorname{dim} \mathfrak{g}-\operatorname{dim} \mathfrak{h}<5$ (см. [6]).

Попытаемся найти необходимые и достаточные свойства алгебры Ли $\mathfrak{g}$ всех векторных полей Киллинга на римановом аналитическом многообразии $M$ и ее стационарной подалгебры $\mathfrak{h}$, при выполнении которых $H$ замкнута в $G$. Чисто алгебраических средств здесь недостаточно. Для исследования проблемы замкнутости стационарной подгруппы $H$ в односвязной группе $G$ привлекается изучение аналитического продолжения локально заданной римановой аналитической метрики. Многообразия, являющиеся аналитическим продолжением произвольной локально заданной римановой аналитической метрики, имеют одну и ту же алгебру Ли всех векторных полей Киллинга. Поэтому вопрос замкнутости группы $H$ в $G$ эквивалентен вопросу об аналитической продолжаемости данной локально заданной римановой аналитической метрики на локально однородном пространстве до метрики полного многообразия. Понятие аналитического продолжения римановой аналитической метрики присутствовало в классических монографиях Хелгасона [4] и С. Кобаяси, Ш. Номидзу [1], но развития не получило.

В $[2,3,7,8]$ изучается, причем не только для римановых многообразий, но также для псевдоримановых пространств и пространств аффинной связности, случай, когда $\mathfrak{g}$ имеет нулевой центр. Доказано, что в этом случае подгруппа $H$, определяемая стационарной подалгеброй $\mathfrak{h}$, будет замкнутой в односвязной группе $G$, порожденной алгеброй $\mathfrak{g}$. Помимо алгебраического подхода, развивается аналитический подход для изучения аналитического продолжения римановых аналитических многообразий. Одной из тем данной работы является исследование локально однородных многообразий, алгебра Ли $\mathfrak{g}$ всех векторных полей Киллинга которых имеет нетривиальный центр $\mathfrak{z}$. Выведены свойства алгебры $\mathfrak{g}$, ее стационарной подалгебры $\mathfrak{h}$ и центра $\mathfrak{z}$, обеспечивающего замкнутость подгруппы $H$, определяемой стационарной подалгеброй $\mathfrak{h}$, в односвязной группе $G$, порожденной алгеброй $\mathfrak{g}$. Пусть $\mathfrak{z}$ - центр алгебры $\mathfrak{g}, \mathfrak{r}-$ ее радикал, а $[\mathfrak{g}, \mathfrak{g}]-$ ее коммутант. Если

$$
\operatorname{dim}(\mathfrak{h} \cap(\mathfrak{z}+[\mathfrak{g}, \mathfrak{g}]))=\operatorname{dim}(\mathfrak{h} \cap[\mathfrak{g}, \mathfrak{g}]),
$$

то $H$ замкнута в $G$. Если для любой полупростой подалгебры $\mathfrak{p} \subset \mathfrak{g}$, удовлетворяющей условию $\mathfrak{p}+\mathfrak{r}=\mathfrak{g}$, где $\mathfrak{r}$ - радикал $\mathfrak{g}$, имеет место соотношение $(\mathfrak{p}+\mathfrak{z}) \cap \mathfrak{h}=\mathfrak{p} \cap \mathfrak{h}$, то $H$ замкнута в $G$.

Принципиальным является исследование случая вполне неоднородной римановой метрики, т.е. метрики, не допускающей никаких движений (полей Киллинга). В этом случае удается определить так называемое квазиполное многообразие, обладающее свойством непродолжаемости и единственности для каждой локально заданной вполне неоднородной метрики (см. [3]). Определение квазиполного многообразия удается обобщить на случай, когда алгебра Ли всех векторных полей Киллинга для заданной локально определенной римановой аналитической метрики не имеет центра (см. [2]). Такое многообразие $M$ обладает свойством максимально возможной симметрии, т.е. любая изометрия $f: U \rightarrow V$ между связными открытыми подмножествами многообразия $M$ аналитически продолжается до изометрии $f: M \rightarrow M$. Однако квазиполное многообразие обладает не только тем недостатком, что оно определено не для произвольной локально заданной метрики, но оно в определенном смысле не является «самым полным». Поэтому далее для произвольной локально заданной римановой метрики мы приведем понятие псевдополного многообразия, исследуем его свойства и связь с квазиполным многообразием, а также опишем псевдополные многообразия в случае малых размерностей. 
2. Аналитическое продолжение римановых многообразий и обобщение понятия полноты. Класс всех локально изометричных римановых аналитических многообразий будем называть также классом многообразий, происходящих из данного ростка риманова аналитического многообразия, а конкретное многообразие из этого класса будем называть аналитическим продолжением данного ростка. Естественным требованием к аналитическому продолжению ростка является непродолжаемость полученного многообразия. Перейдем к точным определениям и формулировкам.

Определение 2.1. Аналитическим продолжением риманова аналитического многообразия $M$ назовем риманово аналитическое многообразие $N$ такое, что существует аналитическое вложение $M$ в $N$ как собственного открытого подмножества. Многообразие, не допускающее аналитического продолжения называется непродолжаемым.

Определение 2.2. Локальной изометрией между двумя римановыми аналитическими многообразиями $M$ и $N$ называется изометрия $\varphi: U \rightarrow V$ между открытыми подмножествами $U \subset M$, $V \subset M$. Многообразия, между которыми существует локальная изометрия, назовем локально изометричными.

Любое векторное поле $X \in \mathfrak{g}$ аналитически продолжается вдоль любой кривой на многообразии $M$, и тем самым алгебра Ли $\mathfrak{g}$ определяет алгебру Ли $\mathfrak{g}$ векторных полей Киллинга на любом односвязном многообразии $N$ локально изометричном $M$. Этот факт верен также для многообразий аффинной связности.

Лемма 2.1. Пусть $M$-аналитическое многообразие афбинной связности, $X$ - инфинитезимальное аффинное преобразование, заданное в области $U \subset M$, и пусть $\gamma(t), 0 \leqslant t \leqslant 1$, такая непрерывная кривая в $M$, что $\gamma(0) \subset U$. Тогда векторное поле аналитически продолжаемо вдоль $\gamma(t)$. Если кривые $\gamma(t)$ и $\delta(t), 0 \leqslant t \leqslant 1, \gamma(0)=\gamma(0), \gamma(1)=\gamma(1)=x_{1}$, гомотопны, то продолжения векторных полей в точку $x_{1}$ вдоль этих кривых совпадают.

Доказательство. Предположим, что $X$ аналитически продолжаемо в окрестность любой точки $\gamma(t)$ при $0 \leqslant t \leqslant t_{1}$. Докажем, что $X$ продолжается и в окрестность точки $q=\gamma\left(t_{1}\right)$. Пусть $V-$ нормальная окрестность точки $q$, являющаяся нормальной окрестностью каждой из своих точек (см. [4]). Рассмотрим $t \leqslant t_{1}$. такое, что $p=\gamma(t) \in V$.

Векторное поле $X$ порождает локальную однопараметрическую группу изометрий $\phi_{s}$ в окрестности каждой точки $\gamma(t), t \leqslant t_{1}$. Докажем, что для всех достаточно малых значений $s$ локальные изометрии $\phi_{s}$ аналитически продолжаются и в окрестность точки $q=\gamma\left(t_{1}\right)$. Тогда векторное поле скоростей этой локальной группы изометрий и будет аналитическим продолжением векторного поля $X$ в окрестность точки $q$.

Рассмотрим связное открытое множество $V_{0} \subset V$, содержащее точки $p$ и $q$, замыкание которого также принадлежит $V, \bar{V}_{0} \subset V, p, q \in V_{0}$. Рассмотрим малую окрестность $V^{\prime} \subset V_{0}$ точки $q$ и соединим точку $p$ отрезком геодезической $\alpha(t), 0 \leqslant t \leqslant 1$, с произвольной точкой $q^{\prime} \in V^{\prime}$. Пусть

$$
Y=\frac{d \alpha}{d t}(0) \in T_{p} M, \quad p_{s}=\varphi_{s}(p), \quad Y_{s}=\varphi_{s}(Y)
$$

Из точки $p_{s}$ проведем геодезическую $\beta(t), 0 \leqslant t \leqslant 1$, такую, что $d \beta / d t=Y_{s}$. При достаточно малых значениях $s \beta(t) \in V_{0}, 0 \leqslant t \leqslant 1$. Положим $\varphi_{s}\left(q^{\prime}\right)=\beta(1)$. Полученное таким образом отображение и есть аналитическое продолжение аффинного преобразования $\varphi_{s}$.

Принципиальным является исследование случая вполне неоднородной римановой метрики, т.е. метрики, не допускающей никаких движений (полей Киллинга). В этом случае удается определить так называемое квазиполное многообразие, обладающее свойством непродолжаемости и единственности для каждой локально заданной вполне неоднородной метрики (см. [2]).

Определение 2.3. Риманово аналитическое многообразие называется вполне неоднородным многообразием, если на нем не существует векторных полей Киллинга. Риманову метрику вполне неоднородного многообразия назовем вполне неоднородной метрикой. 
По лемме 2.1 все многообразия, локально изометричные вполне неоднородному многообразию, являются вполне неоднородными.

Определение 2.4. Вполне неоднородное ориентированное риманово аналитическое многообразие называется квазиполным, если оно непродолжаемо и не допускает нетривиальных сохраняющих ориентацию локальных изометрий в себя.

Приведем основные свойства вполне неоднородных квазиполных многообразий (см. [2]). Для произвольного вполне неоднородного многообразия $M$ рассмотрим множество $S \subset M$ всех неподвижных точек всевозможных сохраняющих ориентацию локальных изометрий многообразия $M$ в себя.

Теорема 2.1. Для произвольного вполне неоднородного риманова аналитического многообразия $M^{\prime}$ множество $S \subset M^{\prime}$ является аналитическим подмножеством коразмерности не меньше чем 2. Следовательно, $M^{\prime} \backslash S$ является связным многообразием.

Теорема 2.2. Для любого вполне неоднородного риманова аналитического многообразия $M^{\prime}$ существует локально изометричное ему квазиполное многообразие $M$ и локально изометрическое накрывающее отображение $f: M^{\prime} \backslash S \rightarrow M$. Таким образом, квазиполное многообразие обладает свойством единственности для каждой вполне неоднородной локально заданной римановой аналитической метрики.

Определение квазиполного многообразия удается обобщить на случай, когда алгебра Ли всех векторных полей Киллинга для заданной локально определенной римановой аналитической метрики не имеет центра (см. [2]). Таковыми являются и многие локально однородные многообразия, в частности все локально симметрические пространства.

Определение 2.5. Риманово аналитическое многообразие $M$ называется локально однородным, если в любой точке $p \in M$ векторные поля Киллинга образуют базис касательного пространства $T_{p} M$.

Эквивалентное определение локально однородного многообразия $M$ состоит в том, что для любых точек $p, q \in M$ существует такая локальная изометрия $\varphi$ многообразия $M$, что $\varphi(p)=q$.

Определение 2.6. Ориентированное риманово аналитическое многообразие, алгебра векторных полей которого имеет нулевой центр, называется квазиполным, если оно непродолжаемо и не допускает нетривиальных сохраняющих ориентацию и все векторные поля Киллинга локальных изометрий в себя.

Исследуем ориентированные римановы аналитические многообразия, алгебра Ли всех векторных полей Киллинга которых не имеет центра, с целью доказать, что каждое такое многообразие локально изометрично квазиполному многообразию, а локально однородное квазиполное многообразие является полным однородным многообразием.

Обозначим через $Z(M)$ псевдогруппу всех сохраняющих ориентацию и векторные поля Киллинга локальных изометрий риманова аналитического многообразия $M: \varphi \in Z(M)$ тогда и только тогда, когда $\varphi X=X$ для любого $X \in \mathfrak{g}$.

Лемма 2.2. Пусть $M-$-риманово аналитическое многообразие, удовлетворяющее свойству однозначного продолэения векторных полей Киллинга и алгебра Ли всех векторных полей Киллинга которого не имеет иентра. Тогда множество $S \subset M$, состоящее из неподвижсних точек всевозможных изометрий $\varphi \in Z(M)$, является аналитическим подмножеством коразмерности не меньше чем 2.

Доказателъство. Докажем, что для любого открытого множества $U \subset M$ с компактным замыканием имеется только конечное число локальных изометрий из $U$ в себя, принадлежащих псевдогруппе $Z(M)$. Предположим противное и рассмотрим бесконечную последовательность локальных изометрий $\varphi_{i} \in Z(M)$, область определения и множество значений которых лежат в $U$. При доказательстве [3, лемма 3] по бесконечной последовательности локальных изометрий $\varphi_{i}$ на 
некотором открытом множестве $V \subset U$ было построено векторное поле Киллинга $X$, которое при переходе к подпоследовательности удовлетворяет следующему условию: для любого $t \in[-1,1]$ существует такое $k(i) \in \mathbb{N}$ так, что

$$
\lim _{i \rightarrow \infty} \varphi_{i}^{k(i)}=\operatorname{Exp} t X
$$

где $\operatorname{Exp} t X$ - локальная однопараметрическая группа изометрий, порожденная векторным полем $X$. Следовательно, для любого векторного поля $Y$ на $V$ существует такое $i \in \mathbb{N}$, что выполняется неравенство

$$
\begin{aligned}
\left|(\operatorname{Exp} t x)_{*} Y-Y\right| \leqslant\left|\varphi_{i}^{k(i)} Y-Y\right|+\mid( & \operatorname{Exp} t X)_{*} Y-\varphi_{i}^{k(i)} Y \mid \leqslant \\
& \leqslant 0+\left|Y-(\operatorname{Exp} t(-X))_{*} \varphi_{i}^{k(i)} Y\right| \leqslant \frac{1}{2}\left|(\operatorname{Exp} t x)_{*} Y-Y\right| .
\end{aligned}
$$

Следовательно, для любого $Y \in \mathfrak{g}$ имеем $(\operatorname{Exp} t X)_{*} Y=Y$, т.е. $[X, Y]=0$. Но это противоречит отсутствию центра в алгебре $\mathfrak{g}$.

Полученное противоречие доказывает существование только конечного числа локальных изометрий из $U$ в $U$, принадлежащих псевдогруппе $Z(M)$. А отсюда, как было показано в [3], уже легко следует тот факт, что множество $S$ является аналитическим подмножеством коразмерности не меньшей чем 2.

В силу леммы 2.2 , многообразие $M \backslash S$ связно.

Лемма 2.3. Пусть $M$-риманово аналитическое многообразие, удовлетворлющее свойству однозначного продолжения векторных полей Киллинга, и алгебра Ли всех векторных полей Киллинга которого не имеет центра. Тогда существует локально изометрическое накрывающее отображение из $M \backslash S$ в риманово аналитическое многообразие $M_{1}$, также удовлетворяющее свойству однозначного продолжения векторных полей Киллинга и псевдогруппа $Z\left(M_{1}\right)$ которого состоит только из тождественного преобразования.

Доказательство. Профакторизуем многообразие $M^{\prime} \backslash S$ по псевдогруппе $Z(M)$. Из доказательства леммы 2.2 следует, что для каждой точки $x \subset M \backslash S$ существует окрестность $U_{1 x} \in M \backslash S$ точки $x$, которая не допускает нетождественных сохраняющих ориентацию локальных изометрий в себя, принадлежащих псевдогруппе $Z(M)$. Это доказывает, что фактор-отображение $\pi$, проектирующее многообразие $M \backslash S$ во множество $M_{1}=M \backslash S / Z(M)$, является накрывающим отображением. Значит, для каждой точки $x \in M$ существует такая ее окрестность $U_{x} \subset M_{1}$ и такое открытое множество $V_{x} \subset \pi^{-1}\left(U_{x}\right)$, что отображение $\pi$ устанавливает гомеоморфизм между множествами $V_{x}$ и $U_{x}$. Определим риманово скалярное произведение. Сузив, если необходимо, множество $V_{x} \subset M \backslash S$, будем считать, что $V_{x}$ является координатной окрестностью точки $y \in \pi^{-1}\left(U_{x}\right) \subset M \backslash S$. Тогда объявим множество $U_{x} \subset M_{1}$ координатной окрестностью точки $x \in M_{1}$. Рассмотрим две такие окрестности $U_{1}, U_{2} \subset M_{1}, U_{1} \cap U_{2} \neq 0$. Заметим, что соответствующие множествам $U_{1}, U_{2}$ множества $V_{1}, V_{2} \subset M \backslash S$ могут и не пересекаться. Положим $\pi^{-1}\left(U_{1} \cap U_{2}\right) \cap V_{1}=V_{10}, \pi^{-1}\left(U_{1} \cap U_{2}\right) \cap V_{2}=V_{12}$. Тогда существует изометрия $\alpha: V_{10} \simeq V_{20}$. Пусть $\psi_{1}$ и $\psi_{2}$ - координатные отображения на $V_{1}$ и $V_{2}$ соответственно. Тогда $\psi_{1} \pi^{-1}$ и $\psi_{2} \pi^{-1}$ будут координатными отображениями на $U_{1}$ и $U_{2}$.

Рассмотрим произвольную точку $x \in M_{1}$ и произвольные векторы $X, Y \in T_{x} M_{1}$. Рассмотрим какую-нибудь точку $\pi^{-1}(x) \subset M \backslash S$ и такие векторы $X_{1}, Y_{1} \in T_{y} M$, что $\pi_{*} X_{1}=X, \pi_{*} Y_{1}=Y$. Определим риманово скалярное произведение $\langle X, Y\rangle$ равным имеющемуся на $T_{x} M$ риманову скалярному произведению $\left\langle X_{1}, Y_{1}\right\rangle$. Если взять другую точку $z \in \pi^{-1}(x)$ и такие векторы $X_{2}, Y_{2} \in$ $T_{z} M$, что $\pi_{*} X_{2}=X, \pi_{*} Y_{2}=Y$, то существует такая локальная изометрия $\varphi \in Z(M)$, что $\varphi(z)=$ $y, \varphi_{*} X_{2}=X_{1}, \varphi_{*} Y_{2}=Y_{1}$, Следовательно, $\left\langle X_{1}, Y_{1}\right\rangle=\left\langle X_{2}, Y_{2}\right\rangle$. Это доказывает корректность определения римановой метрики на $M_{1}$.

Построенное риманово многообразие $M_{1}$ не допускает нетождественных сохраняющих ориентацию локальных изометрий, индуцирующих тождественное преобразование на алгебре векторных полей Киллинга $\mathfrak{g}$. Проекция $\pi: M \backslash S \rightarrow M_{1}$ является локально изометрическим накрывающим 
отображением. Остается доказать свойство однозначного продолжения векторных полей Киллинга на $M_{1}$. Рассмотрим векторное поле Киллинга $X$, заданное на некотором открытом множестве $U \subset M_{1}$, и такие открытые множества $U_{0} \subset U$ и $V_{0} \subset M \backslash S$, что накрывающее отображение $\pi$ устанавливает изометрию между множествами $V_{0}$ и $U_{0}$. Тогда векторное поле $\pi_{*}^{-1} X$ однозначно продолжается с множества $V_{0} \subset M$ на все многообразие $M$ и задает векторное поле $Y$ на $M$. Пусть точки $y, z \in M \backslash S$ таковы, что $\pi(x)=\pi(y)$ и $\pi_{*} Y(z)=\pi_{*} \varphi_{*} Y(y)$. Так как $\pi \varphi=\pi$ по определению $\pi$, то $\pi_{*} \varphi_{*}=\pi_{*}$. Следовательно, $\pi_{*} Y(z)=\pi_{*} \varphi_{*} Y(y)=\pi_{*} Y(y)$. Это доказывает, что отображение $\pi$ однозначно проектирует векторное поле $Y$, заданное на $M$, на векторное поле $\pi_{*} Y$, заданное на многообразии $M_{1}$. Полученное векторное поле $\pi_{*} Y$ и будет аналитическим продолжением векторного поля $X$ на все многообразие $M_{1}$.

Теорема 2.3. Произвольное риманово аналитическое многообразие $M$, алгебра Ли векторных полей Киллинга которого не имеет иентра, локально изометрично квазиполному многообразию.

Доказательство. Рассмотрим произвольное риманово аналитическое многообразие $M^{\prime}$, алгебра Ли векторных полей Киллинга которого не имеет центра. Многообразие $M_{1}$, построенное при доказательстве леммы 2.3, не допускает локальных изометрий в себя, сохраняющих ориентацию и векторные поля Киллинга. Тогда квазиполным многообразием $M$ будет некоторое максимальное аналитическое продолжение многообразия $M_{1}$. Будем считать, что все многообразия, которые мы будем рассматривать при доказательстве теоремы, обладают свойством однозначного аналитического продолжения векторных полей Киллинга, т.е. алгебра Ли всех векторных полей Киллинга одинакова для всех многообразий и равна $\mathfrak{g}$. Если $M^{\prime}$ удовлетворяет этому свойству, то и многообразие $M_{1}$ ему удовлетворяет.

Рассмотрим множество $\Lambda$, состоящее из аналитических продолжений $M_{\alpha}$ многообразия $M_{1}$, удовлетворяющих свойству однозначного продолжения векторных полей Киллинга и не допускающих локальных изометрий, тождественных на алгебре всех векторных полей Киллинга. Снабдим многообразие $M_{1}$ отмеченной точкой и отмеченным репером в отмеченной точке, а образы этой точки и этого репера отметим в многообразиях $M_{\alpha} \in \Lambda$. Введем на этом множестве следующее отношение порядка. $M_{\alpha} \leqslant M_{\beta}$, если существует изометрическое вложение $i_{\alpha \beta}: M_{\alpha} \rightarrow M_{\beta}$, переводящее отмеченную точку в отмеченную и отмеченный репер в отмеченный. В результате $\Lambda$ становится частично упорядоченным множеством. Рассмотрим произвольное линейно упорядоченное подмножество $\Delta$ множества $\Lambda$, Построим прямой предел семейства многообразий $M_{\alpha} \in \Delta$ и отображений $i_{\alpha \beta}$. Получим многообразие $M_{0}$, обладающее следующими свойствами. Для любого многообразия $M_{\alpha} \in \Delta$ существует изометрическое вложение $i_{\alpha}: M_{\alpha} \rightarrow M_{0}$, причем $i_{\alpha}\left(M_{\alpha}\right) \subset i_{\beta}\left(M_{\beta}\right)$, если $M_{\alpha} \leqslant M_{\beta}$. Пусть $M_{0}=\bigcup M_{\alpha}$. Докажем, что $M_{\alpha} \in \Lambda$. Произвольное векторное поле $X$ на многообразии $M_{1}$ при помощи вложений $i_{1 \alpha}: M_{1} \rightarrow M_{\alpha}$ и $i_{\alpha}: M_{\alpha} \rightarrow M_{0}$ переносится на многообразие $i_{\alpha}\left(M_{\alpha}\right) \subset M_{0}$, причем $\left(i_{\alpha} i_{1 \alpha}\right)_{*} X=\left(i_{\beta} i_{1 \beta}\right)_{*} X$ на $i_{\alpha}\left(M_{\alpha}\right) \cap i_{\beta}\left(M_{\beta}\right)$, а векторное поле Киллинга $\left(i_{\alpha} i_{1 \alpha}\right)_{*} X$ однозначно продолжается с подмногообразия $i_{\alpha}\left(M_{\alpha}\right) \subset M_{0}$ на любое подмногообразие $i_{\beta}\left(M_{\beta}\right) \subset M_{0}, M_{\beta} \geqslant M_{\alpha}$, и, значит, на все многообразие $M_{0}$. Таким образом, векторное поле Киллинга, заданное на произвольно малом открытом множестве $U \subset M_{0}$ однозначно продолжается до векторного поля Киллинга на $M_{0}$.

Рассмотрим теперь локальную изометрию $\varphi \in Z(M)$. Пусть точка $x_{0} \in M_{0}$ принадлежит области определения изометрии $\varphi$. Тогда точки $x_{0}$ и $\varphi\left(x_{0}\right)$ лежат в некотором подмногообразии $i_{\alpha}\left(M_{\alpha} \subset M_{0}\right.$. Следовательно, $\varphi \in Z\left(i_{\alpha}\left(M_{\alpha}\right)\right)$, и поэтому $\varphi$ является тождественным преобразованием. Значит, псевдогруппа $Z\left(M_{0}\right)$ состоит только из тождественного преобразования. Итак, для произвольного линейно упорядоченного подмножества $\Delta \subset \Lambda$ мы построили верхнюю грань. По лемме Цорна множество $\Lambda$ имеет максимальный элемент. Мы утверждаем, что многообразие $M$, являющееся таким максимальным элементом, и будет искомым квазиполным многообразием. Требуется доказать, что $M$ непродолжаемо.

Предположим противное и обозначим через $N$ нетривиальное продолжение многообразия $M$. Пусть $S \subset N$ так же, как и выше, обозначает множество неподвижных точек всевозможных локальных изометрий из псевдогруппы $Z(N)$. Точно так же, как и при доказательстве леммы 2.3 
было профакторизовано многообразие $M \backslash S$, профакторизуем многообразие $N \backslash S$. В результате получим многообразие $L$, удовлетворяющее свойству однозначного продолжения векторных полей Киллинга и не допускающего локальных изометрий, сохраняющих ориентацию и все векторные поля Киллинга. Обозначим через $i$ вложение $i: M \rightarrow N$. Докажем, что $i(M) \cap S=\varnothing$. Если $x \in i(M)$, то и некоторый нормальный шар $B$ с центром в $x$ принадлежит $i(M)$. Если, кроме того, $x \in S$, то существует локальная изометрия $\varphi \in Z(M)$, удовлетворяющая условию $\varphi(x)=X$. Эта изометрия определяет изометрию шара $B$ в себя, заданную в нормальных координатах линейным отображением - дифференциалом изометрии $\varphi$. Но существование такой изометрии противоречит тривиальности псевдогруппы $Z(M)$. Таким образом, $i$ дает вложение $i: M \rightarrow N \backslash S$. Сквозное отображение $\pi i: M \rightarrow L$, где $\pi: N \backslash S \rightarrow L-$ построенное при доказательстве леммы 2.3 накрывающее отображение, является также вложением. Так как если $\pi i(x)=\pi i(y)$, то существует такая локальная изометрия $\varphi \in Z(M)$, что $\varphi(x)=\varphi(y)$, следовательно, $x=y$. В силу того, что $M$ - максимальный элемент множества $\Lambda, \pi i$ является изометрией, и $N \backslash S$ накрывает $M$.

Имеем накрывающее отображение $\pi: N \backslash S \rightarrow M$ и вложение $i: M \rightarrow N \backslash S$, причем $i(M)$ открыто в $N \backslash S$. Пусть имеется последовательность точек $x_{n} \in i(M)$, сходящаяся к $x \in N \backslash S$. Тогда последовательность $y_{n}=\pi\left(x_{n}\right)$ также сходится к некоторой точке $y \in M$. Но тогда, так как $x_{n}=i\left(y_{n}\right)$, то $x=i(y) \in M$. Это доказывает замкнутость $i(M)$ в $N \backslash S$. Итак, $N \backslash S$ несвязно или $N \backslash S=N$, Но несвязность $N \backslash S$ противоречит лемме 2.2. Поэтому $N \backslash S=M$. Докажем, что $S=\varnothing$. Предположим противное и рассмотрим нормальный шар $B$ с центром в некоторой точке $x \in S \subset N$. Существует изометрия шара $B$ в себя. Эта изометрия не оставляет неподвижными точки из $B \backslash S$ и поэтому является нетождественной локальной изометрией из псевдогруппы $Z(N \backslash S)$. Но так как $N \backslash S=M$, то это противоречит тривиальности псевдогруппы $Z(M)$. Это доказывает, что $S+\varnothing, N=M$ и $M$ непродолжаемо.

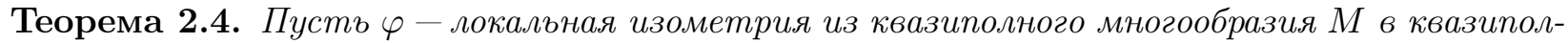
ное многообразие $N$. Тогда $\varphi$ продолжается до изометрии $\varphi: M \simeq N$.

Доказательство. Рассмотрим произвольную точку $X \in M$ и гладкую кривую $\gamma(t), 0 \leqslant t \leqslant 1$, $\gamma(t) \in D(\varphi) \subset M, \gamma(1)=x$. Докажем, что изометрию $\varphi$, заданную в окрестности $U=D(\varphi) \subset M$ точки $x_{0}=\gamma(0)$, можно продолжить вдоль кривой $\gamma$. Предположим, что такого продолжения не существует. Рассмотрим минимальное число $t_{1} \in[0,1]$ среди таких чисел $t$, что изометрия $\varphi$ не продолжается в окрестность точки $\gamma(t)$ вдоль кривой $\gamma$. Докажем, тем не менее, что вопреки предположению продолжение $\varphi$ на некоторую окрестность точки $\gamma\left(t_{1}\right)$ вдоль кривой $\gamma$ существует.

В силу предположения, сделанного относительно $t_{1}$, для любого $t \in\left[0, t_{1}\right)$ изометрия $\varphi$ определена в некоторой окрестности точки $\gamma(t)$. Так что на $N$ определена кривая $\delta(t)=\varphi(\gamma(t))$, $0 \leqslant t \leqslant t_{1}$. Пусть $x_{1}=\gamma\left(t_{1}\right)$ и $\varepsilon>0$ таково, что окрестность $U_{\varepsilon}=\left\{x \in M, \rho\left(x, x_{1}\right) \leqslant \varepsilon\right\}$ является нормальной окрестностью каждой из своих точек. Так как для любого $y \in N$ и любого $\varepsilon_{0}>0$ существует такое $\alpha>0$, что для всех $t^{\prime}, t^{\prime \prime} \in\left[0, t_{1}\right)$, удовлетворяющих условиям $\left|t_{1}-t^{\prime}\right|<\alpha$ и $\left|t_{1}-t^{\prime \prime}\right|<\alpha$, выполняются неравенства

$$
\left|\rho\left(y, \delta\left(t^{\prime}\right)\right)-\rho\left(y, \delta\left(t^{\prime \prime}\right)\right)\right| \leqslant \rho\left(\delta\left(t^{\prime}\right), \delta\left(t^{\prime \prime}\right)\right) \leqslant \int_{t^{\prime}}^{t^{\prime \prime}} \sqrt{\left\langle\delta^{\prime}(t), \delta^{\prime}(t)\right\rangle} d t=\int_{t^{\prime}}^{t^{\prime \prime}}\left\langle\gamma^{\prime}(t), \gamma^{\prime \prime}(t)\right\rangle d t<\varepsilon_{0} .
$$

Следовательно, для любого $y \in N$ существует

$$
\lim _{t \rightarrow t_{1}} \rho(y, \delta(t))=\rho_{1}(y) .
$$

Рассмотрим множество

$$
V_{\varepsilon}=\left\{y \in N \mid \rho_{1}(y)<\varepsilon\right\} .
$$

Существует изометрия $\varphi=\psi^{-1}$ некоторой окрестности $V_{D} \subset V_{\varepsilon}$ множества

$$
D=\left\{y \in N \mid y=\delta(t), t_{2} \leqslant t<t_{1}\right\}
$$

на окрестность $U_{D} \subset U_{\varepsilon}$ множества

$$
B=\left\{x \in M \mid x=\gamma(t), t_{2} \leqslant t<t_{1}\right\} .
$$


Докажем, что $\psi$ можно продолжить до изометрии $\psi: V \simeq V$. Докажем сначала, что $\psi$ вдоль любой кривой $\nu(s), 0 \leqslant s \leqslant 1$ на $V_{\varepsilon}, \nu(0) \in V_{D}, \nu(1)=y$ - произвольная точка на $V_{\varepsilon}$. Если предположить, что это не так, то существует минимальное число $s_{1}$ среди чисел $u \in[0,1]$, обладающих свойством: $\psi$ не продолжается вдоль кривой $\nu(s)$ в какую-нибудь окрестность точки $\nu(u)$. Пусть $\sigma>0$ и $s_{2}<s_{1}$ таковы, что множество

$$
B_{\sigma}=\left\{y \in N \mid \rho\left(y, \nu\left(s_{2}\right)\right)<\sigma\right\}
$$

является нормальной окрестностью точки $\nu\left(s_{2}\right)$ и $\rho\left(\nu\left(s_{2} ; \nu\left(s_{1}\right)\right)<\sigma / 2\right.$. Следовательно, $\nu\left(s_{1}\right) \in B_{\sigma}$. Используя линейность отображения $\psi$ в нормальных координатах, можно продолжить изометрию $\psi$, определенную на некоторой окрестности точки $\nu\left(s_{2}\right)$, до изометрии $\psi$, определенной на всем множестве $B_{\sigma}$, являющимся окрестностью точки $\nu(s)$. Это опровергает предположение о непродолжаемости $\psi$ вдоль кривой $\nu(s)$.

Докажем теперь, что продолжение изометрии $\psi$ вдоль всевозможных кривых на $V_{\varepsilon}$ дает однозначное отображение $\psi: V_{\varepsilon} \rightarrow U_{\varepsilon}$. Предположим противное. Тогда существует такая замкнутая жорданова кривая $\nu(t), 0 \leqslant t \leqslant 1, \nu(0)=\nu(1)$, на $V_{\varepsilon}$, что кривая $\beta(t)=\psi(\nu(t))$ на $U_{\varepsilon}$ будет незамкнутой, $\beta(0) \neq \beta(1)$. Но так как всевозможные аналитические продолжения изометрии $\psi$ индуцируют одинаковые отображения на алгебре векторных полей Киллинга, то изометрия вида $\psi \psi^{-1}$, переводящая $\beta(0)$ в $\beta(1)$, принадлежит псевдогруппе $Z(M)$, а это противоречит тому, что $M$ является квазиполным многообразием. Аналогично доказывается, что продолжение локальной изометрии $\phi=\psi^{-1}$ из $U_{\varepsilon}$ в $V_{\varepsilon}$ задает однозначное отображение на множестве $\varphi\left(V_{\varepsilon}\right) \subset U_{\varepsilon}$. Итак, имеем изометрическое вложение $V_{\varepsilon} \rightarrow U_{\varepsilon}$. Докажем, что оно является сюръективным отображением. Если предположить противное, то, склеив многообразия $N$ и $U_{\varepsilon}$ по отображению $\psi$, получим нетривиальное продолжение многообразия $N$, что противоречит его непродолжаемости. Следовательно, имеем изометрию $\psi: V_{\varepsilon} \rightarrow U_{\varepsilon}$. Обратная изометрия $\psi^{-1}: U_{\varepsilon} \rightarrow V_{\varepsilon}$ дает продолжение изометрии $\psi$ на окрестность $U_{\varepsilon}$ точки $\gamma\left(t_{1}\right)$ вдоль кривой $\gamma$ вопреки первоначальному предположению относительно $t_{1}$.

Таким образом, мы доказали, что локальная изометрия $\varphi$ из $M$ в $N$ продолжается в любую точку $x \in M$ вдоль произвольной кривой на $M$. Точно так же, как выше мы доказали, что продолжение изометрии $\psi$ вдоль всевозможных кривых на $V_{\varepsilon}$ дает взаимно однозначное отображение, определенное на всем $V_{\varepsilon}$, доказывается, что продолжение $\varphi$ вдоль всевозможных кривых на $M$ дает изометрическое вложение $\varphi: M \rightarrow N$.

Следствие 2.1. Произвольное риманово аналитическое многообразие, алгебра Ли векторных полей Киллинга которого не имеет центра локально изометрично единственному квазиполному многообразию. Иными словами, локально заданная риманова аналитическая метрика, алгебра Ли векторных полей Киллинга которой не имеет иентра, единственным образом продолжается до квазиполного многообразия.

Доказательство. Пусть квазиполное многообразие $M$ локально изометрично многообразию $M^{\prime}$ и пусть $N$ - другое квазиполное многообразие, локально изометричное многообразию $M^{\prime}$. Тогда существует локальная изометрия $\varphi$ из $N$ в $M^{\prime}$ и локальная изометрия $\psi$ из $M^{\prime}$ в $M$. Суперпозиция изометрий $\varphi$ и $\psi$ является локальной изометрией из $N$ в $M$. По теореме 2.4 локальная изометрия $\psi \varphi$ продолжается до изометрии $M \simeq N$. Что и требовалось доказать.

Следствие 2.2. Пусть $\mathfrak{g}$ - алгебра Ли всех векторных полей Киллинга риманова аналитического многообразия $M^{\prime}$, диффеоморфном шару, а $\mathfrak{h}-е е$ стачионарная подалгебра. Пусть $G-$ односвязная группа, порожденная алгеброй $\mathfrak{g} u \mathrm{H}-$ ее подгруппа, порожденная подалгеброй $\mathfrak{h}$. Если $\mathfrak{g}$ не имеет иентра, то $H$ замкнута в $G$.

Доказательство. Так как $M^{\prime}$ диффеоморфно шару, его векторные поля Киллинга аналитически продолжаются на нем однозначно. По теореме 2.3 многообразие $M^{\prime}$ локально изоморфно квазиполному многообразию $M$, имеющему ту же самую алгебру Ли $\mathfrak{g}$ всех векторных полей Киллинга и ту же самую стационарную подалгебру $\mathfrak{h}$. Для произвольного векторного поля $X \in \mathfrak{g}$ при всех значениях параметра $t$, меньших некоторого числа $\delta$, элементы однопараметрической 
группы преобразований $\operatorname{Exp} t X$ являются локальными изометриями многообразия $M$. По теореме 2.4 они продолжаются до изометрий всего многообразия $M$. Но тогда определены изометрии $\operatorname{Exp} t X=(\operatorname{Exp} t X)^{n}$. Таким образом, группа $G$ действует на $M$, а $H$ является ее стационарной подгруппой. Это означает, что орбита группы $G$ на $M$ накрывается однородным многообразием $G \backslash H$. Следовательно, $H$ замкнута в $G$.

Отметим, что квазиполное многообразие являются наиболее сжатым, т.е. универсально притягивающим объектом в категории всех локально изометричных многообразий. Для любого риманова аналитического многообразия $M^{\prime}$, алгебра векторных полей Киллинга которого не имеет центра, существует локально изометрическое отображение из $M^{\prime}$ в квазиполное многообразие $M$, определенное на всем $M^{\prime}$.

Квазиполное многообразие единственно в классе всех аналитических продолжений данного ростка и обладает рядом замечательных свойств (см. [3]). Прежде всего, свойством максимальной симметрии, т.е. любая локальная изометрия $f: U \rightarrow V$ из квазиполного многообразия $M$ в себя аналитически продолжается до изометрии $f: M \rightarrow M$. Однако понятие квазиполного многообразия обладает не только тем недостатком, что оно определено не для всех локально заданных римановых аналитических метрик, но оно также не является в определенном смысле «самым полным». А именно, существует росток риманова аналитического многообразия, допускающий продолжение до полного многообразия, каноническое продолжение которого до квазиполного не является полным многообразием.

Пример 2.1. Рассмотрим эллипсоид в трехмерном пространстве, заданный уравнением

$$
\frac{x^{2}}{a^{2}}+\frac{y^{2}}{b^{2}}+\frac{z^{2}}{c^{2}}=1 .
$$

Для того, чтобы получить квазиполное многообразие в классе всех римановых аналитических многообразий, локально изометричных эллипсоиду, необходимо выбросить из эллипсоида 6 точек пересечения с осями координат и профакторизовать полученное многообразие по группе вращений на 180 градусов вокруг всех осей координат.

Дать обобщение понятия полноты, приводящее к «самому полному» многообразию для произвольного ростка риманова аналитического многообразия оказывается возможным.

Определение 2.7. Риманово аналитическое односвязное многообразие $M$ называется псевдополным, если оно обладает следующими свойствами. $M$ непродолжаемо. Не существует локально изометрического накрывающего отображения $f: U \rightarrow M$, где $N$ - односвязное риманово аналитическое многообразие, а $f(M)$ открытое подмножество в $N$, не равное $N$.

Исследуем аналитическое продолжение до псевдополного многообразия для различных классов ростков римановых аналитических многообразий. Прежде всего следует установить тот факт, что аналитическое продолжение до псевдополного многообразия существует для любого ростка риманова аналитического многообразия. Вместе с тем в общем случае это продолжение не единственно, однако различные аналитические продолжения одного и того же ростка различаются не очень значительно.

Теорема 2.5. Любое локально заданное риманово аналитическое многообразие допускает аналитическое продолжение до псевдополного многообразия. Если в классе локально изометричных римановых аналитических многообразий имеется полное многообразие, то это многообразие является единственным псевдополным многообразием в этом классе.

Доказательство. На множестве всех односвязных аналитических продолжений данного ростка риманова аналитического многообразия введем следующее отношение порядка. Многообразие $M$ больше или равно многообразию $N, M \succeq N$, если существует локально изометрическое отображение $f: N \rightarrow M$. Тем самым множество односвязных локально изометричных друг другу римановых аналитических многообразий превращается в частично упорядоченное множество. По лемме Цорна это множество содержит максимальный элемент. Этот элемент по определению и будет псевдополным многообразием. 
Рассмотрим полное риманово аналитическое многообразие $M$. Если предположить, что $M$ не является псевдополным, то существует такое локально изометрическое отображение $f: M \rightarrow N$, что найдется точка $x \notin N$. Пусть $\gamma(t), 0 \leqslant t \leqslant 1$, геодезическая, соединяющая точку $y \in f(M)$ с точкой $x$. Тогда прообраз этой геодезической при $0 \leqslant t \leqslant \delta$ не продолжается до геодезической при всех $t$ на многообразии $M$, что противоречит полноте этого многообразия. Псевдополное многообразие не единственно в классе всех локально изометричных римановых аналитических многообразий.

Пример 2.2. Рассмотрим росток двумерного риманова аналитического многообразия, носителем которого является сфера с метрикой

$$
d s^{2}=\frac{f(z, \bar{z})}{\sqrt{\left(1+|z|^{6}\right.}} d z d \bar{z},
$$

где $f(z, \bar{z})$ - аналитическая функция на сфере, удовлетворяющая условию

$$
f(z, \bar{z}) \neq\left|A^{\prime}(z)\right|^{2} f(A(z), A(\bar{z}))
$$

для любого дробно-линейного преобразования $A(z)$.

Такая метрика имеет особенность в точке $z=\infty$. Сфера с данной метрикой является псевдополным многообразием. Устраним особенность в точке $z=\infty$ при помощи преобразования $z=w^{2}+a, a \in \mathbb{C}$. В результате получим сферу, двулистно накрывающую первоначальную и имеющую метрику

$$
d s^{2}=\frac{4|w| f\left(w^{2}+a, \bar{w}^{2}+\bar{a}\right)}{\sqrt{1+\left|w^{2}+a^{2}\right|^{6}}} d w d \bar{w} .
$$

Эта метрика имеет особенность в точке $w=0$, что является естественным, так как сфера $w$ ветвится над сферой $z$ в точке $z=a$, соответствующей точке $w=0$. При различных $a$ получаем различные псевдополные многообразия с координатой $w$.

Как показывает пример 2.2, имеется большое множество не очень естественных псевдополных многообразий. С целью избежать разветвления над регулярными точками, сузим понятие псевдополного многообразия.

Определение 2.8. Риманово аналитическое односвязное многообразие $M$, называется правильным псевдополным многообразием, если не существует накрывающего локально изометрического отображения $f: M \backslash S \rightarrow N$ в другое псевдополное многообразие $N$ локально изометричное многообразию $M$.

Теорема 2.6. Локальная изометрия из правильного псевдополного многообразия $M$ в псевдополное многообразие $N$ аналитически продолжается вдоль непрерывных кривых в любую точку $M$ за исключением аналитического подмножества $S$ коразмерности не менвше чем 2.

Доказательство. приведем для случая, когда алгебра Ли всех векторных полей Киллинга не имеет центра. Рассмотрим подмножества $S \subset M$ и $S^{\prime} \subset N$, состоящие из всех неподвижных точек локальных изометрий, сохраняющих ориентацию векторных полей Киллинга. Множества $S$ и $S^{\prime}$ являются аналитическими подмножествами многообразий $M$ и $N$ коразмерности не меньшей, чем 2 (см. $[2,3])$. Пусть $M_{0}$ - квазиполное многообразие, локально изометричное многообразиям $M$ и $N$. Тогда существуют накрывающие локально изометрические отображения $f: M \backslash S \rightarrow M_{0}$ и $g: N \backslash S^{\prime} \rightarrow M_{0}$ (см. [2,3]). При этом, из определения правильного псевдополного многообразия следует, что $f(M \backslash S)=M_{0}$ и $g\left(N \backslash S^{\prime}\right)=M_{0}$. Рассмотрим произвольную кривую $\gamma(t) \subset M \backslash S$ такую, что первоначально заданная локальная изометрия $\phi$ между многообразиями $M$ и $N$ содержит точку $\gamma(0)$, ее образ $\delta(t)=f(\gamma(t)) \subset M_{0}$ и связную компоненту $\beta(t)$ прообраза $g^{-} 1((t)) \subset S^{\prime}$, содержащую точку $\phi(\gamma(0))$. Тогда первоначально заданная локальная изометрия $\phi$ аналитически продолжается до изометрии некоторой окрестности кривой $\gamma(t), 0 \leqslant t \leqslant 1$, на некоторую окрестность кривой $\beta(t), 0 \leqslant t \leqslant 1$, принадлежащую $N \backslash S^{\prime}$. 
Для произвольного ориентированного риманова аналитического многообразия $M$ обозначим через $Z(M)$ псевдогруппу, состоящую из всех локальных изометрий многообразия $M$, сохраняющих ориентацию и все векторные поля Киллинга. Рассмотрим фактор-многообразие $K_{M}$ многообразия $M \backslash S$ по псевдогруппе $Z(M)$. Определим объединение многообразий $K_{M}$ и $K_{N}$, склеивая их по множеству $K_{M \cup N}$. Под объединением $M \cup N$ подразумевается отождествление максимальных подмножеств, на которые продолжается первоначально заданная локальная изометрия между односвязными накрывающими $\widetilde{M}$ и $\widetilde{N}$ многообразий $M$ и $N$. На многообразии $M \backslash S$ рассмотрим распределение $\mathfrak{z}^{\perp}$, состоящее из векторов, перпендикулярных центру $\mathfrak{z}$ алгебры Ли $\mathfrak{g}$ всех векторных полей Киллинга.

Теорема 2.7. Росток риманова аналитического многообразия, на носителях которого распределение $\mathfrak{z}^{\perp}$ касательных векторов, перпендикулярных иентру $\mathfrak{z}$ алгебры всех векторных полей Киллинга, инволютивно, допускает аналитическое продолжение до правильного псевдополного многообразия. Если из такого псевдополного многообразия $M$ выбросить множество $S$ неподвиюных точек, сохраняющих ориентацию и все векторные поля Киллинга локальных изометрий, то односвязная накрывающая $\widehat{M \backslash S}$ многообразия $M \backslash S$ изометрична прямому произведению евклидова пространства и односвязной накрывающей $\widetilde{K}$ квазиполного многообразия $K$, локально изометричного вполне геодезическому подмногообразию, касательного $\kappa \mathfrak{z}^{\perp}$. $\widetilde{M \backslash S} \approx \mathbb{R}^{k} \times \widetilde{K}$.

Доказательство. Ввиду инволютивности распределений $\mathfrak{z}$ и $\mathfrak{z}^{\perp}$ некоторая окрестность $U$ отмеченной точки $p \in M$ имеет вид $U=V \times W$, где $V$-открытое подмножество интегрального подмногообразия распределения $\mathfrak{z}$, а $W$-открытое подмножество интегрального подмногообразия распределения $\mathfrak{z}^{\perp}$. Пусть $x^{1}, \ldots, x^{k}$ - координаты на $V$, а $y^{1}, \ldots, y^{m}$-координаты на $W$. Тогда в координатах $x^{1}, \ldots, x^{k}, y^{1}, \ldots, y^{m}$ компоненты $g_{i j}$ не зависят от $x^{1}, \ldots, x^{k}$, и так как подмногообразия $V$ и $W$ перпендикулярны, то компоненты при $d x^{i} d y^{j}$ равны 0. Поэтому метрика на $U$ имеет вид

$$
d s^{2}=d s_{1}^{2}(y)+f_{i j}(y) d x^{i} d x^{j} .
$$

Вследствие непродолжаемости псевдополного многообразия, $M \backslash S$ содержит полные интегральные подмногообразия распределения $\mathfrak{z}$, т.е. прямые произведения евклидова пространства и тора $\mathbb{R}^{s} \times T^{l}$. Поэтому $M \backslash S$ является расслоением над $K^{\prime} \subset K$ со слоями $\mathbb{R}^{s} \times T^{l}$. Так как распределение $\mathfrak{z}^{\perp}$ инволютивно, это расслоение содержит сечение $K^{\prime}$ и поэтому тривиально, $M \backslash S=\mathbb{R}^{s} \times T^{l} \times K^{\prime}$. Так как $M$ непродолжаемо, то $K^{\prime}=K$. Следовательно, односвязная накрывающая многообразия $M \backslash S$ изометрична прямому произведению односвязных пространств, $\widetilde{M / S} \approx \mathbb{R}^{k} \times \widetilde{K}$.

Следствие 2.3. Рассмотрим риманово аналитическое многообразие $M^{\prime}$ размерности $n$, алгебра Ли $\mathfrak{g}$ которого коммутативна, т.е. совпадает со своим иентром $\mathfrak{z}, u \operatorname{dim} \mathfrak{g}=\operatorname{dim} \mathfrak{z}=n-1$. Тогда существует не более двух псевдополных многообразий, локально изометричных $M^{\prime}$.

Доказательство. Так как $\operatorname{codim} \mathfrak{z}=1$, то $\operatorname{dim} \mathfrak{z}^{\perp}=1$, и $\mathfrak{z}^{\perp}$ инволютивно. По теореме 2.5 для псевдополного многообразия $M$ локально изометричного многообразию $M^{\prime}$ имеет место разложение $M \backslash S=\mathbb{R}^{s} \times T^{l} \times K$. Вполне геодезическое подмногообразие $K$ изометрично прямой $\mathbb{R}$ или окружности $S^{1}$ или лучу $(a, \infty)$ или интервалу $(a, b)$. Рассмотрим фактор-множество $\bar{K}=M \backslash Z(M)$. Если $K=\mathbb{R}$ или $K=S^{l}$, то $\bar{K}=K$. Если $K=(a, \infty)$, то $\bar{K}=[a, \infty)$ или $\bar{K}=K=(a, \infty)$. Если $K=(a, b)$, то $\bar{K}=[a, b)$ или $\bar{K}=(a, b]$ или $\bar{K}=[a, b]$ или $\bar{K}=K=(a, b)$.

В случае, если $K=\mathbb{R}$ или $K=S^{1}$, то соответствующий росток риманова аналитического многообразия имеет единственное продолжение до псевдополного многообразия, и это многообразие изометрично евклидову пространству. Продолжение ростка до псевдополного многообразия будет единственным в случае $S=\infty$, т.е. $\bar{K}=K$.

Пусть $K=(a, \infty)$, а $\bar{K}=[a, \infty)$. Тогда точки подмножества $S \subset M$ отображаются при факторизации $\bar{K}=M / Z(M)$ в точку $a \in \bar{K}$. Точка $x \in S$ является особой точкой некоторого поля $X \in \mathfrak{z}, X(x)=0$, а любая изометрия $\varphi$ из $M$ в себя, для которой $\varphi(x)=x$, имеет вид $\phi=\operatorname{Exp} t Y$, 
$Y \in \mathfrak{z}$. Рассмотрим подалгебру $\mathfrak{z} 0 \subset \mathfrak{z}$, состоящую из векторных полей Киллинга $X \in \mathfrak{z}$, обращающихся в ноль в точке $x, X(x)=0$. Тогда $\mathfrak{z}_{0}$ порождает группу изометрий некоторого шара $B$ аналитически продолжающуюся до группы изометрий многообразия $M$ и изоморфную факторгруппе группы $\mathfrak{z}_{0}=\mathbb{R}^{s}$ по некоторой решетке $\Gamma$, действующей на многообразии $M$. Тогда $M$ является полным многообразием, изометричным пространству $\mathbb{R}^{s} \times T^{l}$. Аналогичная конструкция применима к случаю, когда $K=(a, b)$, а $\bar{K}=[a, b)$ или $\bar{K}=(a, b]$, т.е. когда $\bar{K}$ получается из $K$ присоединением одной точки $a$ или $b$. В этом случае псевдополное многообразие также единственно и изометрично многообразию $\mathbb{R}^{s} \times T^{l} \times \bar{K}$, однако это многообразие уже не является полным.

Наконец рассмотрим случай $K=(a, b), \bar{K}=[a, b]$, т.е. когда $\bar{K}$ получается из $K$ присоединением двух точек $a$ и $b$. Рассмотрим псевдополное многообразие $M_{1}$ и точки множества $S_{1} \subset M_{1}$, проектирующиеся в точку $a \in \bar{K}$. Тогда так же, как и при рассмотрении предыдущих случаев, рассмотрим многообразие $M_{1}^{\prime}$, получающееся присоединением множества $S_{1}$ к фактор-многообразию многообразия $M \backslash S$ по некоторой решетке $\left.\Gamma_{1} \subset z=R^{(} n-1\right)$ так, что $M_{1}^{\prime}=\mathbb{R}^{s} \times T^{l} \times \overline{K_{1}}$, где $\overline{K_{1}}=[a, b)$. Аналогично рассмотрим псевдополное многообразие $M_{2}$ и точки множества $S_{2} \subset M_{2}$, проектирующиеся в точку $b \in K$. Многообразие $M_{2}^{\prime}$ получается присоединением множества $S_{2}$ к фактор-многообразию многообразия $M \backslash S$ по некоторой решетке $\Gamma_{2} \subset \mathfrak{z}=R(n-1)$ так, что $M_{2}^{\prime}=\mathbb{R}^{s} \times T^{l} \times \overline{K_{2}}$, где $\overline{K_{2}}=(a, b]$. Если решетки $\Gamma_{1}$ и $\Gamma_{2}$ не совпадают, то многообразия $M_{1}=M_{1}^{\prime}$ и $M_{2}=M_{2}^{\prime}$ являются двумя различными псевдополными многообразиями. Если же решетки $\Gamma_{1}$ и $\Gamma_{2}$ совпадают, то многообразия $M_{1}$ и $M_{2}$ изометричны и определяют полное многообразие $M=M_{1}=M_{2}$.

Перейдем к описанию псевдополных многообразий малых размерностей.

Рассмотрим росток $\mathcal{A}$ двумерного риманова вещественно аналитического многообразия. Алгебра Ли $\mathfrak{g}$ векторных полей Киллинга двумерного многообразия имеет размерность не больше чем 3. Если $\operatorname{dim} \mathfrak{g}=3$, то росток $\mathcal{A}$ является ростком многообразия постоянной кривизны и продолжается до полного многообразия - сферы, плоскости или гиперболической плоскости. Если $\operatorname{dim} \mathfrak{g}=2$, то росток $\mathcal{A}$ является ростком левоинвариантной римановой метрики на двумерной группе Ли, которая и будет продолжением данного ростка до полного многообразия. Случай $\operatorname{dim} \mathfrak{g}=1$ описан в только что доказанном следствии к теореме 2.5.

Рассмотрим вполне неоднородные двумерные римановы аналитические многообразия. Фактор-многообразие $K$, построенное ранее как объединение всех фактор-многообразий, локально изометричных друг другу, по псевдогруппе всех локальных изометрий, сохраняющих все векторные поля Киллинга и ориентацию, является не чем иным, как квазиполным многообразием. Рассмотрим множество $\bar{K}=K \cup T$, получающееся присоединением к многообразию $K$ образов точек $x \in S \subset M_{\alpha}$ при фактор-отображениях $\pi: M_{\alpha} \rightarrow M_{\alpha} / Z\left(M_{\alpha}=\bar{K}_{\alpha} \subset \bar{K}\right.$, заданных на всевозможных аналитических продолжениях $M_{\alpha}$ ростка $\mathcal{A}$. Тогда подмножество $T \subset \bar{K}$ состоит из изолированных точек, и на $\bar{K}$ можно ввести структуру аналитического многообразия. Рассмотрим точку $z_{0} \in T \subset \bar{K}$. Тогда существует достаточно малый шар $U_{0}$ с центром в такой точке $x_{0} \in U_{0}$, что фактор-отображение $\pi: U_{0} \rightarrow \bar{K}$ является факторизацией шара $U_{0}$ по конечной группе вращений с центром в $x_{0} \in U_{0}, \pi\left(x_{0}\right)=z_{0}$. Если $z$-комплексная координата на $U_{0}$, то отображение $\pi$ имеет вид $z \rightarrow w=z^{m} 4$, и метрика на множестве $V_{0}=\pi\left(U_{0}\right) \subset \bar{K}$ имеет вид $d s^{2}=|w|^{(-2(m-1)) / m} d s_{1}^{2}(w, \bar{w})$, где $d s_{1}^{2}(w, \bar{w})$ - аналитическая риманова метрика на шаре $V_{0} \subset \bar{K}$.

Обозначим через $\widetilde{K}$ односвязную накрывающую множества $\bar{K}$. Тогда прообразом $\widetilde{T} \subset \widetilde{K}$ множества $T \subset \bar{K}$ является дискретное множество точек $a_{i} \in \widetilde{K}$. На $\widetilde{K} \backslash \widetilde{T}$ однозначно определяется аналитическая риманова метрика так, что накрытие будет локально изометричным. Тогда метрика в окрестностях точек $a_{i}$ имеет вид

$$
d s^{2}=|w|^{-2(m-1) / m} d s_{1}^{2}(w, \bar{w}) .
$$

Односвязное многообразие $\widetilde{K}$ диффеоморфно комплексной плоскости, кругу или сфере.

Рассмотрим случай, когда $\widetilde{K}$ отождествляется с комплексной плоскостью $\mathbb{C}$. Тогда существует функция $f$, голоморфная на $\widetilde{K} \backslash \widetilde{T}$ и имеющая ветвление порядка $m_{i}$ в точках $a_{i}$. Эта функция 
называется функцией Вейерштрасса, имеющей нули заданного порядка в заданных точках.

$$
f(z)=\prod_{i=1}^{\infty} \sqrt[m_{i}]{1-\frac{z}{a_{i}}} \exp \left(\frac{1}{m_{i}}\left(\frac{z}{a_{i}}+\frac{1}{2}\left(\frac{z}{a_{i}}\right)^{2}+\cdots+\frac{1}{p_{i}}\left(\frac{z}{a_{i}}\right)^{p_{i}}\right)\right),
$$

где числа $p_{i} \in \mathbb{N}$ таковы, что при любом $z \in \mathbb{C}$ сходится ряд

$$
\sum_{i=1}^{\infty}\left(\frac{z}{a_{i}}\right)^{p_{i}}
$$

Рассмотрим риманову поверхность $M$ функции $f(z)$. Поверхность $M$ накрывает комплексную плоскость $\mathbb{C}$ так, что накрывающее отображение $\pi: M \rightarrow \mathbb{C}$ имеет ветвление порядка $m_{i}$ над точками $a_{i} \in \mathbb{C}$ и не разветвлено в остальных точках. Определим риманову метрику на $M$, положив $g(X, Y)=g\left(\pi_{*} X, \pi_{*} Y\right)$, где $X, Y \in T_{x} M, \pi_{*} X, \pi_{*} Y \in T_{\pi(x)} K$. Эта метрика не имеет особенностей в таких точках $x_{i} \in M$, что $\pi\left(x_{i}\right)=a_{i}$. Легко доказать, что для любого односвязного многообразия $N$, локально изометричного $M$, любая локальная изометрия $\phi$ из $N$ в $M$ аналитически продолжается до локально изометрического отображения $\phi: N \rightarrow M$. Таким образом, $M$ является единственным аналитическим продолжением данного ростка до псевдополного многообразия.

В случае, если $\widetilde{K}$ - круг, то, аналогично случаю комплексной плоскости, строится единственное аналитическое продолжение данного ростка до псевдополного многообразия. Это многообразие также является римановой поверхностью голоморфной функции $f(z)$ на $\widetilde{K}$, имеющей ветвление порядка $m_{i}$ над точками $a_{i} \in \widetilde{T} \subset \widetilde{K}$.

$$
f(z)=\prod_{i=1}^{\infty} \sqrt[m_{i}]{\frac{z-a_{i}}{z-\alpha_{i}}} \exp \sum_{k=1}^{q_{i}} \frac{\left(a_{i}-\alpha_{i}\right)^{k}}{k\left(z-\alpha_{i}\right)^{k}}
$$

где $\alpha_{i}$ - точка на границе круга, ближайшая к $a_{i}$, а числа $q_{i} \in \mathbb{N}$ таковы, что

$$
\left|\ln \frac{z-a_{i}}{z-\alpha_{i}}+\sum_{k=1}^{q_{i}} \frac{\left(a_{i}-\alpha_{i}\right)^{k}}{k\left(z-\alpha_{i}\right)^{k}}\right| \leqslant \frac{1}{2^{i}} .
$$

Рассмотрим случай, когда $\widetilde{K}$ - сфера. Здесь множество $\widetilde{T} \subset \widetilde{K}$ состоит из конечного числа точек $\alpha_{0}, \alpha_{1}, \ldots, \alpha_{l}$, в каждой из которых метрика имеет особенность вида

$$
d s^{2}=|w|^{-2(m-1) / m} d s_{1}^{2}(w, \bar{w}) .
$$

Функция

$$
f(z)=\prod_{i=1}^{\infty} \sqrt[m_{i}]{\frac{z-a_{i}}{z-\alpha_{0}}}
$$

на сфере имеет ветвление порядка $m_{i}$ в точках $a_{i}, i=1,2, \ldots, l$ и ветвление порядка $m$ в точке $\alpha_{0}$. Так же, как и выше, рассмотрим риманову поверхность $M$ функции $f(z)$. Накрывающее отображение $\pi: M \rightarrow \widetilde{K}=S^{2}$ является накрытием над $\widetilde{K} \backslash \widetilde{T}$ и имеет ветвление порядка над точками $a_{i} \in \widetilde{T} \subset \widetilde{K}, i=1,2, \ldots, l$ и ветвление некоторого порядка $m$ в точке $\alpha_{0}$. Тогда метрика на $M$, индуцированная метрикой на $\widetilde{K}$ и накрывающим отображением $\pi$, не имеет особенности в точках $\pi^{-1}\left(a_{i}\right)$, но в случае, если $m \neq m_{0}$, имеет особенность в точке $\pi^{-1}\left(a_{0}\right)$. Полученное многообразие является правильным псевдополным многообразием. Вместо точки $a_{i} \in \widetilde{T} \subset \widetilde{K}$ можно взять любую другую точку $a_{j} \in \widetilde{T} \subset \widetilde{K}$ и построить описанным выше способом другое правильное псевдополное многообразие. Таким образом, мы получим все аналитические продолжения до правильного многообразия данного ростка.

Перейдем к описанию трехмерных псевдополных многообразий. По-прежнему будем обозначать через $\mathfrak{z}$ центр алгебры Ли $\mathfrak{g}$ всех векторных полей Киллинга на рассматриваемых многообразиях. Если $\operatorname{dim} \mathfrak{z}=3$, то росток риманова многообразия является однородным, и, согласно результату Мостова (см. [6]), продолжается до однородного многообразия. Если $\operatorname{dim} \mathfrak{z}=2$, то 
согласно доказанному следствию из теоремы 2.5, существует не более двух аналитических продолжений данного ростка до правильного псевдополного многообразия. Случай, когда алгебра $\mathfrak{g}$ не имеет центра, $\operatorname{dim} \mathfrak{z}=0$, разобран при доказательстве теоремы 2.4 .

Рассмотрим случай, когда $\operatorname{dim} \mathfrak{z}=2$. Сначала рассмотрим случай, когда $\widetilde{K}$ диффеоморфно плоскости. Рассмотрим многообразие $M_{0} \approx \widetilde{K} \times \mathfrak{z}$. Пусть $U_{0}$ - малый шар, снабженный изначально заданной римановой метрикой, $V_{0}=U_{0} / Z\left(U_{0}\right) \subset \widetilde{K}$. Распространим метрику, заданную на $U_{0}$, на многообразие $V_{0} \times \mathfrak{z}$. Пусть $x^{1}, x^{2}, x^{3}$ - такие координаты на $V_{0} \times \mathfrak{z}$, что $x^{1}, x^{2}$-координаты на $V_{0}$, а $x^{3}$ - координата на $\mathfrak{z}$. Компоненты метрического тензора $g_{i j}\left(x^{1}, x^{2}\right)$ не зависят от $x^{3}$. Функции $g_{i j}\left(x^{1}, x^{2}\right)$ аналитически продолжаются вдоль любой кривой на $\widetilde{K}$ и задают метрику на $M_{0} \approx \widetilde{K} \times \mathfrak{z}$. Тогда $M_{0} \backslash Z\left(M_{0}\right)=K$, следовательно, $Z\left(M_{0}\right)=K \times \Gamma$, где $\Gamma$ - группа накрытия $\widetilde{K} \rightarrow K$. Тогда для правильного псевдополного многообразия $M$ многообразие $M / S=K \times \mathfrak{z} / \Gamma_{0}$, где $\Gamma_{0}$ - дискретная подгруппа группы $\mathfrak{z}$.

Рассмотрим теперь случай, когда фактор-многообразие $K$ диффеоморфно сфере. Разобьем $K$ в объединение двух открытых дисков $K=K_{1} \cup K_{2}$. Построим, как и выше, римановы многообразия $M_{1}=K_{1} \times \mathbb{R}$ и $M_{2}=K_{2} \times \mathbb{R}$, являющиеся аналитическими продолжениями первоначально заданного ростка, у которых подмногообразия $\mathbb{R}$ являются интегральными кривыми векторного поля $X \in \mathfrak{z}$. Локальные изометрии $f$ из $M_{1}$ в $M_{2}$ продолжаются вдоль любой кривой на $\left(K_{1} \cap K_{2}\right) \times \mathbb{R}$. Если такое продолжение однозначно, получим полное многообразие $M \approx S^{2} \times \mathbb{R}$, являющееся продолжением заданного ростка. Предположим теперь, что существует замкнутая кривая $\gamma(t), 0 \leqslant t \leqslant 1$, на $\left(K_{1}\right.$ ก $\left.K_{2}\right) \times \mathbb{R}$, продолжение изометрии $f$ вдоль которой неоднозначно, $f(\Gamma(0))=y_{1} \neq y_{2}=f(\gamma(1))$. Пусть $x^{1}, x^{2}, x^{3}$ - такие координаты на $M_{1}$, что $x^{1}, x^{2}$ образуют координаты на $K_{1}$, а $x^{3}$ - координата на $\mathbb{R}$, а $y^{1}, y^{2}, y^{3}$-такие координаты на $M_{2}$, что $y^{1}, y^{2}$ образуют координаты на $K_{2}$, а $y^{3}$ - координата на $\mathbb{R}$. Так как $x^{3}$ и $y^{3}$ являются координатами на алгебре Ли $\mathfrak{z}$, то изометрия $f$ в координатах $x^{1}, x^{2}, x^{3}, y^{1}, y^{2}, y^{3}$ имеют вид $y^{1}=y^{1}\left(x^{1}, x^{2}\right)$, $y^{2}=y^{2}\left(x^{1}, x^{2}\right), y^{3}=x^{3}+f\left(x^{1}, x^{2}\right)$, где функции $y^{1}, y^{2}$ являются функциями перехода от карты $K_{1}$ к карте $K_{2}$ на сфере и поэтому однозначны. А функция $f\left(x^{1}, x^{2}\right)$ неоднозначно продолжается вдоль замкнутой кривой $\delta(t), 0 \leqslant t \leqslant 1$ на $K_{1} \cap K_{2}$. Пусть $f(\delta(1))-f(\delta(0))=a \in \mathbb{R}$. Рассмотрим окружность $S^{1}=\mathbb{R} / \mathbb{Z}$. Тогда продолжение функции $f$ вдоль кривой $\delta$ будет однозначно, если считать, что $f$ принимает значения не на прямой $\mathbb{R}$, а на окружности $S^{1}$. Тогда также однозначно будет продолжение функции $f$ вдоль кривых $\delta^{n}, n \in \mathbb{Z}$. Но, поскольку любая кривая на $K_{1} \cap K_{2}$ гомотопна кривой $\delta^{n}$, продолжение функции $f: U_{0} \rightarrow S^{1}$ вдоль всевозможных кривых однозначно на $K_{1} \cap K_{2}$. В таком случае функция $f$ является функцией перехода расслоения на окружности над сферой $S^{2}$, и мы имеем компактные линзовые пространства в качестве аналитических продолжений заданного ростка.

3. Локально однородные многообразия, алгебра векторных полей Киллинга которых имеет нетривиальный центр. Исследуем случай, когда алгебра $\mathfrak{g}$ имеет ненулевой центр $\mathfrak{z}$ и укажем свойства алгебр $\mathfrak{g}, \mathfrak{h}$ и $\mathfrak{z}$, обеспечивающие замкнутость подгруппы $H$ в $G$.

Определим локальную группу локальных изометрий. Рассмотрим произвольное риманово аналитическое многообразие $M$, алгебру Ли $\mathfrak{g}$, состоящую из векторных полей Киллинга на нем и группу Ли $G$ с алгеброй Ли g. Под локальной группой $G$ (chunk of a group) будем подразумевать малую окрестность единицы группы $G$. Она состоит из локальных изометрий многообразия $M$. Алгебра Ли $\mathfrak{g}$, как правило, не порождает группы изометрий многообразия $M$, но порождает псевдогруппу локальных изометрий. Локальную группу будем обозначать той же буквой, что и группу. Орбита локальной группы локальных изометрий многообразия $M$ является локально однородным многообразием $N$. Заметим также, что локальная группа $H$, порожденная стационарной подалгеброй $\mathfrak{h}$, образует группу изометрий некоторого шара с центром в отмеченной точке многообразия $M$.

Изучим сначала некоторые свойства локальной группы локальных изометрий с точки зрения абстрактных групп преобразований. Рассмотрим локальную группу $G$ как подгруппу группы локальных диффеоморфизмов многообразия $M$ с отмеченной точкой, $G \subset \operatorname{Diff} M$, Назовем элемент $\tilde{n} \in G \subset \operatorname{Diff} M$ умножением справа, если существует такой элемент $n \in G$, что для всех таких 
$x \in M$, что $x=g(e), \tilde{n}(x)=g n(e)$. Умножение справа на элемент $n$ определено корректно, если для любого $h \in H$ существует такой $h_{1} \in H$, что для любой локальной изометрии $g \in G$ выполняется равенство $g h n=g n h_{1}$. Другими словами, $n$ принадлежит нормализатору $N(H)$ группы $H$ в $G$. Обозначим через $N$ локальную группу, состоящую из элементов $n \in G$, умножение справа на которые в группе $G$ порождают локальные изометрии многообразия $M$, а через $\mathfrak{n}$ - ее алгебру Ли. Тогда $h \triangleleft \mathfrak{n} \subset G$. Заметим, что сами умножения справа, т.е. элементы $\tilde{n}$, а также элементы центра $Z$ локальной группы $G$ принадлежат $N$. Обозначим через $M_{0}$ орбиту локальной группы $N$ на $M$. Присоединенное действие элементов $n \in N, g \mapsto n^{-1} g n$, задает локальные изометрии на $M_{0}$.

Найдем подгруппу $G_{0} \subset G$, состоящую из «умножений слева». Рассмотрим отображение $f$ из группы $G$, заданной как группа преобразований множества $G$ в себя, определенное по формуле $f(g)=g(e)=g e$, где $e$ - тождественная локальная изометрия. Тогда, так как $\tilde{n}(e)=e n=n$, то будем считать, что $f(\tilde{n})=n$. На множестве $f(G)$ определим умножение $g_{1} g_{2}=g_{1}(e) g_{2}(e)$. Так определенное умножение превращает $f(G)$ в подгруппу $G_{0} \subset G$. Левые умножения $g \in G_{0}$ дополняются правыми умножениями $\tilde{n}$, т.е. любой элемент $g \in G \subset \operatorname{Diff} G$, для которого $g(x)=g x$ при всех $x \in G$, представим в виде $g=g_{0} \tilde{n}, g_{0} \tilde{n}(x)=g x n$ при всех $x \in G$. Следовательно, $G=G_{0} N, \mathfrak{g}=\mathfrak{g}_{0}+\mathfrak{n}$.

Теорема 3.1. Пусть $\mathfrak{g}$ - алгебра Ли всех векторных полей Киллинга на локально однородном римановом аналитическом многообразии $M . \mathfrak{h}$ - ее стационарная подалгебра. Пусть $G-$ односвязная группа, порожденная алгеброй $\mathfrak{g}$ u $H$-ее подгруппа, порожденная подалгеброй $\mathfrak{h}$. Пусть $\mathfrak{z}$ - иентр алгебры $\mathfrak{g}$, а $[\mathfrak{g}, \mathfrak{g}]-$-е коммутант. Если $\mathfrak{h} \cap(\mathfrak{z}+[\mathfrak{g}, \mathfrak{g}])=\mathfrak{h} \cap[\mathfrak{g}, \mathfrak{g}]$, то в $G$.

Доказательство. Предположим противное. Рассмотрим замыкание $\bar{H}$ группы $H$ в $G$ и подалгебpy $\overline{\mathfrak{h}} \subset \mathfrak{g}$ подгруппы $\bar{H} \subset G$. Подалгебра $\mathfrak{h}$ является нормальной подалгеброй алгебры $\overline{\mathfrak{h}}$ (см. [5]). Будем считать, что для отмеченной точки $p \in M X \in \mathfrak{h} \Longleftrightarrow X(p)=0$. Рассмотрим однопараметрическую подгруппу $\bar{h}_{t} \in \bar{H}, \bar{h}_{t} \notin H$, определяемую векторным полем $\bar{X} \in \overline{\mathfrak{h}}, \bar{X} \notin \mathfrak{h}$. Как доказано в [5], существует тор $T$ в такой простой компактной подгруппе $P \in G$, что $H \cap T$ является всюду плотной обмоткой тора $T$. Поэтому можно считать, что $\overline{\mathfrak{h}}_{t} \in T \subset P$. Тогда векторное поле Киллинга $\bar{X}$ касательных векторов к орбитам локальной однопараметрической группы $\bar{h}_{t}$ принадлежит алгебре $\mathfrak{t}$ группы $T$ и, следовательно, $\bar{X} \in \mathfrak{t} \subset \mathfrak{p}$, где $\mathfrak{p}$-алгебра Ли группы $P$. Существует такие окрестность единицы $U$ в группе $G$ и шар $B_{\delta}$ радиуса $\delta$ с центром в отмеченной точке $p \in M$, что все элементы $g \in U$ группы $G$ определяют локальные изометрии из шара $B_{\delta}$ в шар $B_{2 \delta}$ радиуса $2 \delta$ с центром в $\in M$. Так как элементы $\bar{h}_{t}$ принадлежат замыканию $\bar{H}$ группы $H$ в $G$, то для каждого малого $t$ внутренний автоморфизм $x \mapsto \bar{h}_{t} x \bar{h}^{-1}$ группы $G$ является пределом последовательности внутренних автоморфизмов $x \mapsto h_{n} x h_{n}^{-1}, h_{n} \in H$. При малых $t$ и больших $n$ эти автоморфизмы определяют локальные изометрии из шара $B_{\delta}$ в шар $B_{2 \delta}$.

Все умножения справа коммутируют с умножениями слева, т.е. с элементами группы $G_{0}$, однако могут не коммутировать друг с другом. Докажем, что локальная изометрия $\bar{h}_{t}$ со всеми правыми умножениями. Для этого докажем, что действие элемента $\bar{h}_{t}$ в группе внутренних автоморфизмов группы $G, g \mapsto \bar{h}_{t}^{-1} g \bar{h}_{t}$, задает тождественное отображение на $M_{0}$. Рассмотрим последовательность $h_{n} \in H$, сходящуюся к $\bar{h}_{t}$. Так как $H$ является нормальным делителем в $N$, то $n h_{n}=h_{n} n h_{n}^{\prime}$, где $h_{n}^{\prime} \in H$, то $n H=h_{n}^{-1} n h_{n} H$. Следовательно, внутренние автоморфизмы $g \mapsto h_{n}^{-1} g h_{n}$ индуцируют тождественное отображение на $M_{0}$. Переходя к пределу, получим, что внутренний автоморфизм $g \mapsto \bar{h}_{t}^{-1} g \bar{h}_{t}$ индуцируют тождественное отображение на $M_{0}$.

Так как векторное поле $X$, порождающее локальную однопараметрическую группу $\bar{h}_{t}$, принадлежит компактной подалгебре алгебры $\mathfrak{g}$, то $X$ принадлежит коммутанту $[\mathfrak{g}, \mathfrak{g}]$ алгебры $\mathfrak{g}$.

Векторное поле $Z$ касательных векторов к орбитам локальной однопараметрической группы $z_{t}$ умножений справа на $\bar{h}_{t}$ является векторным полем Киллинга. Докажем, что $Z$ коммутирует со всеми другими векторными полями Киллинга на $M$, т.е. $Z \in \mathfrak{z}$. Так как для любого $n \in \mathfrak{n}$ элементы $\bar{h}_{t} n$ и $n \bar{h}_{t}$ индуцируют одну и ту же локальную изометрию на $M_{0}$, то $\bar{h}_{t}$ индуцируют тождественное отображение на алгебре $\mathfrak{n}$. Следовательно, векторное поле Киллинга $Z$ (так же, 
как и $X)$ принадлежит центру алгебры $\mathfrak{n}$. Для любых элементов $g_{0} \in G_{0}$ и $\tilde{n} \in \tilde{\mathfrak{n}}$, рассматриваемых как автоморфизмы группы $G$, выполняются следующие равенства. $g_{0} \tilde{n}(x)=g_{0}(x n)=g_{0} x n$, $\tilde{n} g_{0}(x)=\left(g_{0} x\right) n=g_{0} x n$. Өто означает, что алгебры $\tilde{\mathfrak{n}}$ и $\mathfrak{g}_{0}$ коммутируют друг с другом. Следовательно, векторное поле $Z$ коммутирует с алгеброй $\mathfrak{g}_{0}$ и с алгеброй $\mathfrak{g}_{0}+\mathfrak{n}$, т.е. $Z \in \mathfrak{z}$.

Tак как $z_{t} \bar{h}_{t}^{-1} H=\bar{h}_{t}^{-1} H \bar{h}_{t}$, то

$$
\operatorname{Exp}(t Z) \operatorname{Exp}(-t X)=z_{t} \bar{h}_{t}^{-1} \in H .
$$

Следовательно, $Z-\bar{X} \in H$. Кроме того, $\bar{X} \in \mathfrak{p}=[\mathfrak{p}, \mathfrak{p}] \subset[\mathfrak{g}, \mathfrak{g}]$. Можно доказать, что $Z \notin[\mathfrak{g}, \mathfrak{g}]$. Тогда $Z-\bar{X} \notin[\mathfrak{g}, \mathfrak{g}]$, т.е.

$$
Z-\bar{X} \in \mathfrak{h} \cap(\mathfrak{z}+[\mathfrak{g}, \mathfrak{g}]) .
$$

С другой стороны, так как $Z-\bar{X} \in \mathfrak{h}$, а $Z-\bar{X} \notin[\mathfrak{g}, \mathfrak{g}]$, то

$$
Z-\bar{X} \in \mathfrak{h} \cap(\mathfrak{z}+[\mathfrak{g}, \mathfrak{g}]), \quad Z-\bar{X} \notin \mathfrak{h} \cap[\mathfrak{g}, \mathfrak{g}]
$$

Тогда векторное поле $Z-\bar{X}$ является стационарным, но не принадлежит коммутанту. Следовательно, $\mathfrak{h} \cap(\mathfrak{z}+[\mathfrak{g}, \mathfrak{g}]) \neq \mathfrak{h} \cap[\mathfrak{g}, \mathfrak{g}]$.

Теорема 3.2. Пусть $\mathfrak{g}$ - алгебра Ли всех векторных полей Киллинга на локально однородном

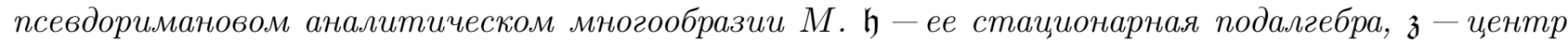
алгебры $\mathfrak{g}, \mathfrak{r}$ - ее радикал. Пусть $G$-односвязная подгруппа, порожденная алгеброй $\mathfrak{g} u$ H ее подгруппа, порожденная подалгеброй $\mathfrak{h}$. Тогда, если для любой полупростой алгебры $\mathfrak{p} \subset \mathfrak{g}$, удовлетворяющей условию $\mathfrak{p}+\mathfrak{r}=\mathfrak{g}$, имеет место равенство $(\mathfrak{p}+\mathfrak{z}) \cap \mathfrak{h}=\mathfrak{p} \cap \mathfrak{h}$, то Н замкнута в $G$.

Доказательство. Предположим противное и рассмотрим замыкание $\bar{H}$ группы $H$ в $G$. Так же, как и при доказательстве теоремы 2.5, рассмотрим однопараметрическую подгруппу $z_{t}$, порожденную умножением справа на элементы однопараметрической группы локальных изометрий $\bar{h}_{t}$ в группе $G$. Пусть $\bar{X}$ - векторное поле Киллинга касательных векторов к орбитам локальной однопараметрической группы локальных изометрий $\bar{h}_{t}^{-1}$, а $Z$ - векторное поле Киллинга локальной однопараметрической группы локальных изометрий $z_{t}$.

Пусть $\mathfrak{p}$ - полупростая подалгебра алгебры $\mathfrak{g}$, содержащая векторное поле $\bar{X}, \bar{X} \in \mathfrak{p} \subset \mathfrak{g}$. Докажем, что $Z+\bar{X} \in \mathfrak{h}$ и $Z+\bar{X} \notin \mathfrak{p}$. В односвязной группе Ли $G$ рассмотрим радикал $R$ (подгруппу, соответствующую подалгебре $\mathfrak{r}$ ) и полупростую подгруппу $P$, соответствующую подалгебре $\mathfrak{p}$. Тогда $R$ - нормальный делитель группы $G, \mathfrak{r}$ - нормальный делитель алгебры $\mathfrak{g}, R \cap P=e, \mathfrak{r} \cap \mathfrak{p}=0$, и имеет место разложение Леви-Мальцева $G=R P$.

Группа $G$ содержит открытую окрестность единицы (chunk of a group), действующую как локальная группа локальных изометрий в окрестности отмеченной точки $p \in M$. Так как $z_{t}$ принадлежит центру группы $G R$, то $z_{t} \in R$, и так как подгруппа $H$ является нормальным делителем группы $\bar{H}$ (см. [4]), то

$$
\bar{h}_{t}^{-1} z_{t} H=\bar{h}_{t}^{-1} H \bar{h}_{t}=H \text {. }
$$

Следовательно, локальные изометрии $\bar{h}_{t}^{-1} z_{t}$ оставляют точку $p$ неподвижной и поэтому принадлежат стационарной подгруппе $H$. Так как $\bar{X} \in \mathfrak{p}$, а $Z \notin \mathfrak{p}$, то $(Z+\bar{X}) \notin \mathfrak{p}$. Поскольку $(Z+\bar{X}) \in \mathfrak{h}$, то доказанное означает, что для выбранной максимальной полупростой алгебры $\mathfrak{p}$ справедливо утверждение $(\mathfrak{p}+\mathfrak{z}) \cap \mathfrak{h} \neq \mathfrak{p} \cap \mathfrak{h}$. Что и доказывает теорему от противного.

4. Заключение. В заключение обозначим задачи, связанные с обобщением полноты риманова аналитического многообразия, требующие своего решения. Во первых, хотелось бы окончательно привести необходимые и достаточные условия замкнутости стационарной подгруппы группы локальных изометрий на римановом многообразии. Причем эти условия должны быть выражены в локальных терминах, т.е. как свойства алгебры Ли всех векторных полей Киллинга. Кроме того, необходимо развить теорию обобщенно полных многообразий для случая существования нетривиального центра в алгебре Ли всех векторных полей Киллинга. В частности, дать обобщение квазиполного многообразия в общем случае. Также желательно более подробно описать 
псевдополные многообразия в общем случае и для конкретных римановых метрик. Условия замкнутости стационарной подгруппы. приведенные в теоремах 3.1 и 3.2, являются необходимыми и «почти достаточными». Хорошо бы найти необходимые и достаточные условия замкнутости стационарной подгруппы в односвязной группе, определяемой алгеброй Ли всех векторных полей Киллинга.

\section{СПИСОК ЛИТЕРАТУРЫ}

1. Кобаяси Ш., Номидзу К. Основания дифференциальной геометрии. Т. 1. - М.: Наука, 1981.

2. Попов В. А. Аналитическое продолжение локально заданных римановых многообразий// Мат. заметки. - 1984. - 36, № 4. - С. 559--570.

3. Попов В. А. Продолжаемость локальных групп изометрий// Мат. сб. - 1988. - 135 (177), № 1. C. $45-64$.

4. Хелгасон С. Дифференциальная геометрия и симметрические пространства. - М.: Мир, 1964.

5. Malcev A. I. On the theory of Lie groups in the large// Мат. сб. - 1945. - 16 (5), № 2. - С. 163-190.

6. Mostow G. D. Extensibility of Local Lie groups of transformations and groups on surfaces// Ann. Math. 1950. - 52. - P. 606-636.

7. Popov V. A. On the extendability of locally defined isometries of a pseudo-Riemannian manifolds// J. Math. Sci. - 2016. - 217, № 5. - P. 624-627.

8. Popov V. A. On Closeness of Stationary Subgroup of Affine Transformation Groups// Lobachevskii J. Math. - 2017. - 38, № 4. - P. 724--729.

Попов Владимир Александрович

Финансовый университет при Правительстве Российской Федерации, Москва

E-mail: vlapopov@gmail.com 